\title{
Abordagens psicoterápicas no transtorno bipolar
}

Psychoterapeutic Approach in Bipolar Disorder

\author{
Paulo Knapp ${ }^{1}$ \\ LUCIANO ISOLAN ${ }^{2}$
}

\begin{abstract}
Resumo
Embora o tratamento farmacológico seja essencial para o tratamento do transtorno bipolar, apenas $40 \%$ de todos os pacientes que aderem às medicações permanecem assintomáticos durante o período de seguimento, o que tem levado ao desenvolvimento de intervenções psicoterápicas associadas. O objetivo deste artigo é examinar as evidências atuais da eficácia de intervenções psicoterápicas no tratamento do transtorno bipolar. Foi realizada uma pesquisa bibliográfica por meio do MedLine, PsychoINFO, Lilacs e Cochrane Data Bank, até o ano de 2004, em que foram procurados artigos originais e revisões sobre as abordagens psicoterápicas utilizadas no tratamento do transtorno bipolar. Há várias abordagens que podem se mostrar úteis no tratamento do transtorno bipolar. A psicoeducação e a terapia cognitivo-comportamental apresentam as evidências mais consistentes e são as técnicas mais amplamente estudadas. As intervenções envolvendo familiares e a terapia interpessoal e de ritmo social se mostram tratamentos eficazes em determinadas situações. Há alguns estudos empregando a terapia psicodinâmica no transtorno bipolar, mas são estudos com limitações metodológicas. Apesar de haver evidências demonstrando a eficácia de determinadas abordagens psicoterápicas no transtorno bipolar, ainda é necessária a realização de estudos posteriores que comprovem tais dados e que desenvolvam tratamentos baseados em modelos etiológicos e que identifiquem tratamentos específicos para as diferentes fases e tipos de transtorno bipolar.
\end{abstract}

Palavras-chave: Transtorno bipolar; psicoterapia; tratamento. 


\begin{abstract}
Although pharmacological treatment is essential for treating bipolar disorder, less than half of all medication compliant patients are non-symptomatic during follow-up, which has led to developments of adjunctive psychosocial interventions. This paper examines the current evidence for effectiveness of psychotherapeutic interventions in the treatment of bipolar disorder. Searches were undertaken through MedLine, PsychoINFO, Lilacs, and Cochrane Data Bank, up to the year 2004. Psychotherapeutic approaches to the treatment of bipolar disorders were searched in original and review articles. Various approaches are useful in the treatment of bipolar disorders. Psychoeducation and cognitive-behavioral therapies show the best available evidence and are the most studied techniques. Family interventions and interpersonal and social rhythm therapy show efficacy in particular treatment phases. Studies with psychodynamic psychotherapies showed methodological limitations. Although there are some current evidence for effectiveness of psychosocial interventions for bipolar disorders, there is still a need for further studies to confirm these data. There is also a need to develop treatments based on etiological models, and particular treatments for the different phases and types of the bipolar spectrum.
\end{abstract}

Key words: Bipolar disorder; psychotherapy; treatment.

\section{Introdução}

O transtorno bipolar pode ser adequadamente tratado com várias classes de medicação, incluindo lítio, anticonvulsivantes, antipsicóticos, antidepressivos e mesmo a eletroconvulsoterapia. Porém, mesmo utilizando-se as mais adequadas estratégias medicamentosas, o curso do transtorno bipolar é, freqüentemente, caracterizado por sintomas crônicos e por altos índices de recaídas e internações. Além disso, mesmo com a remissão dos episódios de humor, ainda podem persistir sintomas subsindrômicos substanciais, principalmente sintomas depressivos, em grande parte dos pacientes.

A compreensão do transtorno bipolar tem sido focada, principalmente, nos aspectos genéticos e biológicos e a psicofarmacoterapia tem-se constituído como um componente essencial do manejo desse transtorno. Porém, há evidências crescentes que sugerem que o curso do transtorno bipolar pode ser modificado por meio de abordagens psicoterápicas. Aimportância de se combinar a psicoterapia com medicações reside no fato de que apenas $60 \%$ dos pacientes bipolares respondem ao lítio ou aos outros estabilizadores do humor. Além do mais, apenas $40 \%$ dos pacientes permanecem sem recaídas/recorrências durante períodos de seguimento de dois a três anos, mesmo em uso de doses adequadas de medicação.

As abordagens psicoterápicas no tratamento do transtorno bipolar têm como objetivos principalmente o aumento da adesão ao tratamento, a redução dos sintomas residuais, a identificação de pródromos sindrômicos com a conseqüente prevenção das recaídas/recorrências, a diminuição das taxas e períodos de hospitalizações e a melhora na qualidade de vida dos pacientes e de seus familiares. Tais abordagens também podem aumentar o funcionamento social e ocupacional desses pacientes e as capacidades de manejarem situações estressantes em suas vidas.

O objetivo deste trabalho é revisar as diferentes abordagens psicoterápicas utilizadas no tratamento do transtorno bipolar, encontradas na literatura.

\section{Material e métodos}

Foi realizada uma pesquisa bibliográfica, pelo Medline, PsychoINFO, Lilacs e Cochrane Data Bank, até o ano de 2004, em que foram procurados artigos originais e revisões sobre as abordagens psicoterápicas utilizadas no tratamento do transtorno bipolar, buscando-se os seguintes termos MeSH: "bipolar disorder", "manic-depressive disorder" e "psychotherapy". Também foram revisadas as referências bibliográficas dos principais artigos pesquisados com o objetivo de localizar artigos que não foram encontrados por meio da busca eletrônica.

\section{Resultados}

Foram encontradas basicamente cinco intervenções psicoterápicas que têm sido utilizadas no tratamento de pacientes com transtorno bipolar: psicoeducação, terapia cognitivo-comportamental, terapia interpessoal e de ritmo social, terapia familiar e conjugal, e terapia psicodinâmica. A seguir serão relatadas mais detalhadamente essas diferentes abordagens psicoterápicas. 


\section{Psic oeduc ação}

Um dos principais objetivos da psicoeducação é a adesão à medicação. Embasada no modelo médico biopsicossocial, a psicoeducação objetiva dar aos pacientes informações sobre a natureza e o tratamento do transtorno bipolar, provendo ensinamentos teóricos e práticos para que o paciente possa compreender e lidar melhor com a sua doença. Outros tópicos abordados em intervenções psicoeducacionais incluem a identificação precoce de sintomas prodrômicos, a coibição de drogas de abuso e o manejo de situações provocadoras do estresse e da ansiedade, entre outros.

Foram encontrados diversos estudos de psicoeducação no tratamento do transtorno bipolar. Num estudo com uma amostra de 60 pacientes realizado por Peet e Harvey (1991), um grupo de 30 pacientes recebeu a intervenção que consistia de materiais explicativos sobre o lítio e uma visita domiciliar, observando-se que, ao final do tratamento, os pacientes que receberam a intervenção, comparados ao do grupo controle em lista de espera, apresentavam melhoras no conhecimento a respeito de sua doença e sobre seu tratamento e maior adesão à medicação, e que tais resultados se mantinham após três meses. Esses dois autores (Van Gent e Zwart, 1991) já haviam conduzido um estudo controlado com cônjuges de pacientes com transtorno bipolar, demonstrando um aumento de conhecimento sobre a doença e seu tratamento após cinco sessões psicoeducacionais em grupo. Nesse estudo, porém, nenhum impacto sobre adesão à medicação foi observado.

Bauer e McBride (1996) desenvolveram um programa estruturado de terapia em grupo utilizando psicoeducação em combinação com algumas estratégias cognitivo-comportamentais e interpessoais para diminuir sintomas e para superar as limitações sociais e ocupacionais dos pacientes. Em um estudo que utilizou essa abordagem, foi observado um maior aumento na adesão medicamentosa (Bauer et al., 1998). Perry et al. (1999) avaliaram a eficácia de um tratamento de sete sessões, que consistia em intervenções individuais que visavam ao manejo dos sintomas e ao reconhecimento de fatores de risco na prevenção de recaídas. Quando comparados a um grupo randomizado para um tratamento controle padrão, os pacientes que receberam psicoeducação apresentaram redução significativa no risco de desenvolverem episódios maníacos e melhora no funcionamento social e ocupacional.

Um recente estudo desenvolvido por Colom et al. (2003) realizou um ensaio clínico que avaliou 120 pacientes com transtorno bipolar tipo I ou tipo II, que estavam em remissão por pelo menos seis meses, e verificou que a psicoeducação realizada em 21 sessões em grupo esteve significativamente associada, em comparação com o grupo controle que recebeu tratamento em grupo não-diretivo, com uma redução no número de recorrências e com um aumento no tempo em que o paciente permanecia livre de sintomas durante o período de tratamento e durante o período de seguimento de dois anos. A quantidade e a duração das hospitalizações também foram menores nos pacientes que receberam psicoeducação. Um estudo (Dogan e Sabanciogullari, 2003) com 26 pacientes bipolares em uso de lítio verificou que a intervenção psicoeducacional em grupo realizada em três sessões demonstrou, ao final de três meses, que os pacientes que receberam a intervenção apresentavam aumento no conhecimento sobre a doença e sobre as medicações, maior adesão às medicações e aumento nos níveis de qualidade de vida, comparados ao grupo que não recebeu esta intervenção.

Há boas evidências para sugerir que as intervenções de psicoeducação, individuais ou em grupo, associadas à farmacoterapia, podem ser promissoras para o tratamento de pacientes bipolares. Mesmo intervenções breves que enfatizam a adesão à medicação e a identificação precoce dos sintomas podem ser benéficas na prevenção de novos episódios de humor e em períodos mais prolongados de eutimia. Porém, pacientes que aderem às medicações e que estão cientes de seus sintomas prodrômicos provavelmente se beneficiarão de outras intervenções com abordagens mais amplas.

\section{Terapia cognitivo-comportamental}

A terapia cognitivo-comportamental (TCC) é uma terapia breve e estruturada, orientada para a solução de problemas, que envolve a colaboração ativa entre o paciente e o terapeuta para atingir objetivos estabelecidos. A terapia é geralmente utilizada no formato individual, embora técnicas de grupo tenham sido desenvolvidas e testadas. Os objetivos da TCC no transtorno bipolar são: 1) educar pacientes e familiares sobre o transtorno bipolar, seu tratamento e suas dificuldades associadas à doença; 2) ensinar métodos para monitorar a ocorrência, a gravidade e o curso dos sintomas; 3) facilitar a aceitação e a cooperação no tratamento; 4) oferecer técnicas não-farmacológicas para lidar com sintomas e problemas; 5) ajudar o paciente a enfrentar fatores estressantes que estejam interferindo no tratamento; 6) estimular a aceitação da doença; 7) aumentar o efeito protetor da família e 8) diminuir o trauma e o estigma associado à doença.

A TCC tem sido a abordagem psicoterápica mais amplamente estudada no transtorno bipolar. Vários estudos evidenciam a eficácia dessa técnica no tratamento de pacientes com transtorno bipolar, incluindo os citados a seguir. O primeiro estudo controlado avaliando a TCC no transtorno bipolar foi realizado por Cochran (1984), no qual foram avaliados 28 pacientes bipolares, comparando TCC individual com o tratamento usual. Cochran utilizou uma abordagem que visava basicamente alterar cognições e comporta- 
mentos que interferissem na adesão medicamentosa. Verificou-se que os pacientes que receberam TCC apresentaram taxas mais altas de adesão e menores taxas de hospitalizações ao término do tratamento de seis semanas e após um seguimento de seis meses. Zaretsky et al. (1999) compararam o efeito de 20 sessões de TCC adaptada para depressão bipolar em 11 pacientes com depressão bipolar em uso de estabilizadores de humor com 11 controles com transtorno depressivo maior que recebiam TCC padrão. Houve diminuição significativa nos sintomas depressivos em ambos os grupos. Fava et al. (2001) avaliaram a TCC em 15 pacientes que recaíram apesar de estarem usando medicação. O tratamento consistiu de dez sessões de 30 minutos a cada semana, que focava no tratamento dos sintomas residuais e incluía psicoeducação, reestruturação cognitiva à terapia de exposição para os sintomas depressivos, ansiosos e para irritabilidade. Esse tratamento se mostrou eficaz no tratamento de sintomas residuais e aumentou o tempo de remissão da doença.

Lam et al. (2000) realizaram um dos primeiros estudos controlados avaliando a TCC em 25 pacientes com transtorno bipolar. Nesse estudo piloto verificouse que a TCC apresentava, em comparação com o tratamento usual, uma diminuição significativa de episódios bipolares durante um período de 12 meses. Um recente ensaio clínico realizado por Lam et al. (2003) analisou 103 pacientes com transtorno bipolar tipo I que apresentavam recaídas freqüentes, apesar da farmacoterapia adequada randomizados para TCC ou para tratamento usual. O tratamento cognitivocomportamental consistiu de 14 sessões nos primeiros seis meses e duas sessões adicionais nos seis meses seguintes. Em um período de seguimento de 12 meses, os pacientes que realizaram TCC apresentaram, significativamente, menos episódios de humor, menos dias em um episódio de humor bipolar, menos hospitalizações, menos sintomas subsíndrômicos, lidaram melhor com pródromos maníacos e apresentam melhor funcionamento social. No seguimento de dois anos do mesmo ensaio clínico (Lam et al., 2005) não foi encontrado efeito significativo na redução de recaídas, embora o grupo que recebeu terapia cognitiva apresentou, novamente, significativa redução em número de dias de episódios de humor bipolar, com melhora significativa nas escalas de humor, no funcionamento social, nas estratégias de enfrentamento dos pródromos de depressão e mania, e nas atitudes interpessoais disfuncionais.

Um estudo controlado, desenvolvido por Scott et al. (2001), avaliou 42 pacientes com transtorno bipolar tipo I e tipo II e demonstrou que a TCC diminuía, significativamente, os sintomas depressivos mas não os sintomas maníacos, sugerindo que a TCC seria particularmente útil para a depressão bipolar. Nesse estudo também verificaram-se aumento da adesão, redução no número de hospitalizações e melhora no funcionamento global.

Há dois estudos abertos evidenciando a eficácia da TCC em grupo no transtorno bipolar (Palmer et al., 1995; Patelis-Siotis et al., 2001). Patelis-Siotis et al. (2001) avaliaram 49 pacientes em TCC, em grupo com sete a 12 integrantes, tratados com 14 sessões semanais de duas horas de duração, e verificaram uma melhora no funcionamento social e na qualidade de vida. Palmer et al. (1995) avaliaram a TCC em grupo em quatro pacientes durante 17 sessões e observaram melhoras no "bem-estar" e nos sintomas depressivos.

Portanto, segundo a literatura científica pesquisada, os estudos têm demonstrado a eficácia da TCC no tratamento do transtorno bipolar, tanto na configuração individual como grupal.

\section{Terapia interpessoal e de ritmo social}

A observação de que muitos pacientes com transtorno bipolar apresentam menos oscilações de humor quando mantêm um modo regular nas suas atividades diárias (sono, alimentação, atividade física) levou ao desenvolvimento de uma psicoterapia chamada de terapia interpessoal e de ritmo social (TIP/RS). ATIP/RS, além das técnicas utilizadas na terapia interpessoal, inclui a psicoeducação sobre o transtorno bipolar e uma abordagem estruturada para a normalização dos ritmos sociais, que são os padrões pessoais esperados de atividades e estimulação social. A hipótese de estabilidade de ritmo social postula que a estabilidade do humor seja, em parte, uma função da regularidade de ritmos sociais e os efeitos desta estabilidade sobre os ritmos circadianos. A TIP/RS objetiva regularizar e padronizar os ritmos diários do paciente e, ao mesmo tempo, resolver áreas-chave de problemas interpessoais-chave que afetam os seus estados de humor e a sua estabilidade de ritmos diários.

Até o momento há dois ensaios clínicos (Frank et al., 1997 e 1999), que utilizaram essa técnica no tratamento no transtorno bipolar. O primeiro estudo (Frank et al., 1997) demonstrou que pacientes que foram tratados com TIP/RS apresentaram maior estabilidade dos ritmos sociais e dos ciclos sono/vigília em comparação com pacientes que não receberam essa mesma abordagem. Porém, um estudo posterior desse mesmo grupo (Frank et al., 1999) não demonstrou diferenças nas taxas de recidiva ou de remissão dos episódios depressivos ou maníacos durante um ano de tratamento. Frank et al. (2000) tem sugerido que, embora não tenha efeitos nem no tempo de remissão nem nas taxas de recaídas, a TIP/RS tem impacto, principalmente, nos sintomas depressivos subsindrômicos.

Os resultados preliminares desses estudos evidenciam que a TIP/RS é uma abordagem que pode ser promissora quando associada ao tratamento farmacológico no transtorno bipolar, porém ainda carecem estudos que comprovem de fato sua eficácia. 


\section{Terapia familiar e de casal}

As intervenções com familiares de pacientes com transtorno bipolar têm como principais objetivos: 1) modificar as interações familiares que interferem no tratamento; 2) psicoeducação para o pacientes e para os seus familiares sobre o transtorno bipolar; 3) desenvolvimento de habilidades de comunicação e 4) desenvolvimento de habilidades de resolução de problemas.

Alguns estudos utilizaram o conceito de expressividade emocional para medir a melhora nos relacionamentos intra-familiares e conjugal. $\mathrm{O}$ índice de expressividade emocional (EE) consiste de três atitudes dos familiares: 1) crítica (expressões de descontentamento ou aborrecimento, acompanhados de um tom de voz negativo); 2) hostilidade (expressões indicando rejeições à pessoa do paciente) e 3) superenvolvimento emocional (extremamente superprotetores, com preocupações excessivas, atitudes de muito sacrifício e renúncia em favor do paciente). A ocorrência em grau elevado de qualquer um desses atributos, em pelo menos um familiar, classifica a família como tendo alta EE, o que está associado a um pior prognóstico, comparados a pacientes com baixa EE.

Estudos avaliando o papel da EE entre familiares de pacientes com esquizofrenia indicam que um alto nível ambiental de EE está associado a um maior risco de recaídas e que intervenções dirigidas à família que reduzem os níveis de EE têm um impacto significativo na redução dos níveis de recaída. Estudos posteriores investigaram se tais achados encontrados em pacientes com esquizofrenia também se aplicariam a pacientes com transtorno bipolar (Miklowitz e Holdstein 1990; Honig et al., 1997; Miklowitz et al., 2000). Um estudo preliminar com nove pacientes bipolares que haviam recentemente tido alta hospitalar por um episódio de mania receberam tratamento psicoeducacional focado na família durante um período de nove meses. Apenas $11 \%$ recaíram, comparados com $61 \%$ de um grupo naturalístico de controles históricos. Honig et al. (1997) compararam uma intervenção familiar psicoeducacional com um grupo controle, que permaneceu em lista de espera, e verificaram que $75 \%$ dos familiares que tinham altos índices de EE passaram a apresentar baixos índices de EE após o tratamento. Porém, não houve mudanças nos níveis sintomáticos após essa intervenção.

Recentemente, Miklowitz et al. (2000) realizaram um ensaio clínico randomizado com 101 pacientes com transtorno bipolar e com seus familiares. Os pacientes do grupo experimental foram tratados com 21 sessões de uma hora, realizadas na casa do paciente, durante um período de nove meses, e eram avaliados trimestralmente por um ano. Durante o período de seguimento de um ano, pacientes que receberam tratamento psicoeducacional focado na família apresentaram taxas significativamente mais baixas de recaídas e mais tempo livre de sintomas do que o grupo controle que recebeu uma breve psicoeducação e intervenções em crises. Esse estudo demonstra a eficácia de intervenções psicoeducacionais focadas na família no tratamento de pacientes com transtorno bipolar.

Existem também estudos abordando terapia com casais no tratamento do transtorno bipolar (Davenport et al., 1977; Clarkin et al., 1998). Davenport et al. (1977) dividiram, não randomicamente, 65 pacientes bipolares eutímicos, que haviam sido recentemente internados para participar de um dos três tipos de tratamento a seguir: 1) terapia de casal mais manejo da medicação; 2) manejo da medicação apenas; 3) atendimento usual. Os pacientes que, junto com seus cônjuges, participaram da terapia de casal apresentaram melhor funcionamento social e menos hospitalizações em um seguimento de dois a dez anos. Clarkin et al., (1998) realizaram um tratamento psicoeducacional para os pacientes bipolares e para suas esposas. Quarenta e dois pacientes foram randomizados para 11 meses de tratamento usual ou para tratamento usual mais 25 sessões de tratamento envolvendo o casal. Este último grupo apresentou taxas significativamente mais elevadas de funcionamento psicossocial e de adesão à medicação do que o grupo que recebeu apenas tratamento usual. Esses estudos sugerem a eficácia de intervenções dirigidas ao casal no tratamento do transtorno bipolar.

\section{Terapia psicodinâmica}

As abordagens psicodinâmicas do paciente bipolar variam conforme as características de maior ou menor integração do paciente no período entre as crises, seu grau de motivação e seu desejo de compreender-se melhor na relação consigo mesmo e com o transtorno bipolar. Como se sabe, essas psicoterapias não se dirigem a sintomas específicos e, no máximo, buscam o significado e a função simbólica destes. Pressupõem, por outro lado, que efeitos benéficos possam ser obtidos pelo esclarecimento de processos intrapsíquicos que podem desencadear ou perpetuar as oscilações de humor em pacientes vulneráveis. Espera-se que tais conflitos e mecanismos de defesa associados se tornem conscientes e que isso permita que dificuldades emocionais estressantes possam ser antecipadas e manejadas, ou que os conflitos sejam resolvidos por meio de insights progressivos. Loeb e Loeb (1987) constataram a utilidade da associação de carbonato de lítio com psicanálise ou psicoterapia de orientação analítica para uma série de pacientes bipolares pelo fato de que tais abordagens permitiam aos pacientes tornarem-se conscientes do ressurgimento de fantasias sexuais e desejos anteriores ao início do episódio maníaco que serviriam como um sinal para a necessidade de aumento da dose de lítio.

Os estudos que avaliaram a psicoterapia psicodinâmica individual sugerem que essa abordagem, associada à medicação, pode ser eficaz no tratamento 
do transtorno bipolar, porém são todos estudos baseados em relatos de caso, com limitações metodológicas e com desfechos clínicos subjetivos. Os estudos em grupo realizados com pacientes bipolares, além das abordagens psicodinâmicas, utilizavam também intervenções ativas que visavam à compreensão da doença e à adesão à medicação. Todos foram estudos abertos e, com exceção de um estudo (Cerbone et al., 1992), não utilizaram avaliadores independentes. Wulsin et al. (1988) avaliaram 28 pacientes que participaram em média de 44 sessões em grupo por um período de 4,5 anos cujo foco era "o efeito da doença nos relacionamentos interpessoais". Os autores concluíram que a terapia em grupo foi bastante factível e benéfica para os pacientes. No estudo de Cerbone et al. (1992), que foi baseado na revisão de prontuários, foram avaliados 43 pacientes bipolares que participaram de um tratamento em grupo que enfocava a psicoeducação e a resolução de problemas interpessoais. Esse estudo verificou que o tratamento em grupo esteve associado com melhoras no funcionamento social e com menor gravidade e duração dos episódios de humor. Retzer et al. (1991), avaliando 20 pacientes com transtorno bipolar e 10 pacientes esquizofrênicos, demonstraram uma diminuição da recorrência de episódios de humor em um período de seguimento de três anos, comparado ao período de pré-tratamento e uma mudança no ponto de vista sobre a doença nos pacientes e nos familiares. Os outros estudos também demonstraram desfechos positivos subjetivos como "bem-estar" (Hallensleben, 1994) e no aprendizado sobre a doença, assim como conviver com ela. Tais estudos, assim como outras abordagens em grupo, sugerem que essas técnicas podem ter um impacto positivo no curso do transtorno bipolar, porém, deve-se ter cautela na interpretação desses dados já que se tratam de estudos com deficiências metodológicas como dados não controlados e avaliação feita por avaliadores não independentes.

\section{Conclusões}

Embora o tratamento farmacológico seja essencial no tratamento do transtorno bipolar, ainda há uma substancial quantidade de pacientes que, apesar da correta adesão à medicação, permanecem sintomáticos. Todos os estudos descritos, independentemente da abordagem utilizada, sugerem que a psicoterapia deve ser utilizada em associação com o tratamento farmacológico. As intervenções psicoterápicas apresentam vários benefícios que incluem diminuição na freqüência e na duração dos episódios de humor, aumento da adesão à medicação, diminuição nas recaídas e impressões clínicas de melhoras gerais.

Há evidências que comprovam que determinadas intervenções têm diferentes efeitos nas distintas fases do transtorno bipolar. A psicoeducação tem se mostrado eficaz e deveria ser utilizada precocemente no curso da doença, porém há algumas dúvidas da sua utilização em pacientes que já são aderentes à medicação. Intervenções com familiares têm demonstrado resultados promissores, principalmente a terapia focada na família desenvolvida por Miklowitz et al. (2000), na prevenção de recaídas, porém, a aplicação dessas técnicas em pacientes com limitadas redes familiares pode ser complicada. A TIP/RS parece ser uma abordagem promissora, principalmente nos sintomas depressivos residuais, no entanto, mais estudos são necessários. Os estudos envolvendo os tratamentos psicodinâmicos sugerem que essa abordagem possa ter algum benefício no transtorno bipolar, porém, são estudos com deficiências metodológicas e tais achados devem ser vistos com cautela e necessitam de confirmação em estudos posteriores. A TCC tem se mostrado a abordagem psicoterápica com mais evidências consistentes de eficácia no tratamento de pacientes bipolares, revelando-se eficaz em vários desfechos significativos do transtorno bipolar. Entretanto, ainda não se sabe se essa psicoterapia é superior a outras abordagens psicoterápicas e qual a dose psicoterápica e quais as técnicas cognitivas e comportamentais que, de fato, são mais eficazes.

As abordagens psicoterápicas deveriam ser individualizadas e utilizadas precocemente no tratamento do transtorno bipolar para melhorar a adesão medicamentosa e ajudar o paciente a identificar os pródromos da doença com o objetivo de aprender a desenvolver estratégias para lidar melhor com tais situações, além de terem efeitos nos sintomas residuais os quais estão associados à cronicidade e a altos níveis de sofrimento e incapacitação.

A pesquisa avaliando o papel da psicoterapia no tratamento do transtorno bipolar ainda está em fase muito inicial, se comparada aos estudos com esquizofrenia. No tratamento da esquizofrenia, uma recente metanálise de 26 ensaios clínicos randomizados de TCC individual e em grupo evidencia claramente a eficácia dessas abordagens (Pilling et al., 2002), mas o papel da TCC no tratamento do transtorno bipolar ainda é uma área que carece de mais estudos.

Várias questões deveriam ser levadas em consideração em estudos futuros, como: 1) a padronização de critérios diagnósticos e de medidas de desfecho para que comparações apropriadas entre diferentes estudos possam ser feitas; 2) a utilização de abordagens psicoterápicas específicas para as diferentes fases e estágios do transtorno bipolar; 3) a avaliação das abordagens psicoterápicas em diferentes tipos de transtorno bipolar (tipo I, tipo II, misto, ciclador rápido); 4) utilização de amostras mais significativas e de ensaios clínicos randomizados que comparem diferentes abordagens psicoterápicas e 5) desenvolvimento de novas abordagens que levem em conta modelos etiológicos do transtorno bipolar. 
Referências bibliográficas

BAuer, M.S.; McBride, L. - Structure group Psychotherapy for Bipolar Disorder. The Life and Goals Program. New York: Springer, 1996.

BAUeR, M.S.; McBride, L.; ChASE C., et al. - Manual-based Group Psychotherapy for Bipolar Disorder: a Feasibility Study. J Clin Psychiatry 59: 449-455, 1998.

Cerbone, M.J.A.; Mayo J.A.; Cuthberston, B.A. et al. - Group Therapy as an Adjunct to Medication in the Management of Bipolar Affective Disorder. Group 16: 174-187, 1992.

Clarkin, J.F.; Carpenter, D.; Hull, J. et al. - Effects of Psychoeducational Intervention for Married Patients with Bipolar Disorder and their Spouses. Psychiatr Serv 49: 531-533, 1998.

Cochran, S. - Preventing Medication Non-compliance in the Outpatient Treatment of Bipolar Affective Disorder. Journal of Nervous \& Mental Diseases 176: 457-464, 1984.

Colom. F.; Vieta, E.; Martinez-Aran, A. et al. - A Randomized Trial on the Efficacy of Group Psychoeducation in the Prophylaxis of Recurrences in Bipolar Patients Whose Disease is in Remission. Arch Gen Psychiatry 60: 402407, 2003.

DavenPort, Y.; Ebert, M.; Adland, M.; et al. - Couples Therapy as an Adjunct to Lithium Maintenance of the Manic Patient. Journal of Orthopsychiatry, 47, 495-502, 1977.

Dogan, S.; Sabanciggullari, S. - The Effects of Patient Education in Lithium Therapy on Quality of Life and Compliance. Arch Psychiatr Nurs 17(6): 270-275, 2003.

Fava, G.A.; Bartoluccl, G.; Rafanelli, C.; Mangell, L. - CognitiveBehavioral Management of Patients with Bipolar Disorder who Relapsed while on Lithium Prophylaxis. Clin Psychiatry 62: 556-559, 2001.

Frank, E.; Hiastala, S.; Ritenour, A. et al. - Inducing Lifestyle Regularity in Recovering Bipolar Disorder Patients: Results from the Maintenance Therapies in Bipolar Disorder Protocol. Biol Psychiatry 41: 1165-1173, 1997.

Frank, E.; Swartz, H.; Mallinger. A. et al. - Adjunctive Psychotherapy for Bipolar Disorder Effects of Changing Treatment Modality. J Abnorm Psychol 108: 579-587, 1999.

Frank, E.; Swartz, H.A.; Kupfer, D.J. - Interpersonal Ans Social Rhytm Therapy: Managing the Caos of Bipolar Disorder. Biol Psychiatry 48: 593-604, 2000.

Hallensleben, A. - Group Psychotherapy with Manic Depressive Patients on Lithium: Ten Years Experience. Group Anal. 27, 475-482, 1994.

Honig, A.; Hofman, A.; Rozendaal, N.; Dingemans, P. - Psychoeducation in Bipolar Disorder: Effect of Expressed Emotion. Psychiatry Res 72: 17-22, 1997.
Lam, D.; Bright, J.; Jones, S. et al. - Cognitive Therapy for Bipolar Illness - a Pilot Study Relapse Prevention. Cognit Ther Res 24: 503-520, 2000.

Lam, D.; Hayward, P.; WatKins, E. et al. - Relapse Prevention in Patients with Bipolar Disorder: Cognitive Therapy Outcome After 2 Years. Am J Psychiatry 162:324-329, 2005.

Lam, D.; Watkins, E.; HaYwARd, P. et al. - A Randomized Controlled Study of Cognitive Therapy for Relapse Prevention for Bipolar Affective Disorder: Outcome of the First Year. Arch Gen Psychiatry 60: 145-152, 2003.

LoEB, F.F.; LoEB, L.R. - Psychoanalytic Observations on the Effect of Lithium on Manic Attacks. J Am Psychoanal Assoc 35: 877-902, 1987.

MıkLoWITZ, D.J.; Goldstein, M.J. - Behavioral Family Treatment for Patients with Bipolar Affective Disorder. Behav Modif 14: 457-489, 1990.

Miklowitz, D.J.; Simoneau, T.L.; George, E.L. et al. - Familyfocused Treatment of Bipolar Disorder: 1 Year Effects of Psychoeducational Program in Conjunction with Pharmacotherapy. Biol Psychiatry 48: 582-592, 2000.

Palmer, A.G.; Willians, H.; Adams, M. - CBT in a Group Format for Bipolar Affective Disorder. Behav Cogn Psychother 23: 153-168, 1995.

Patelis-Siotis, I.; Young, L.T.; RobB, J.C. et al. - Group Cognitive Behavioral Therapy for Bipolar Disorder: a Feasibility and Effectiveness Study. J Affect Disord 65: 145-153, 2001.

Peet, M.; Harvey, N. - Lithium Maintenance: 1. A Standard Education Programme for Patients. British Journal of Psychiatry 158: 197-200, 1991.

Perry, A.; Tarrier, N.; Morris, R. et al. - Randomized Controlled Trial of Efficacy of Teaching Patients with Bipolar Disorder to Identify Early Symptoms of Relapse and Obtain Treatment. BMJ 318: 149-153, 1999.

Pilling, S.; Bebilngton, P.; Kuipers, E. et al. - Psychological Treatments in Schizophrenia: 1.Meta-Analysis of Family Intervention and Cognitive Behaviour Therapy. Psychol. Med. 32, 763-782, 2002.

Retzer, A.; Simon, F.B.; Weber, G., Stierlin, H.M.; Schmidt, G. - A Follow-up Study of Manic Depressive and SchizoAffective Psychoses after Systemic Family Therapy. Family Process. 30, 139-153, 1991.

Scott, J.; Garland, A.; Moorhead, S.A. - Pilot Study of Cognitive Therapy for Bipolar Disorders. Psychol Med 31: 459467, 2001.

Van Gent, E.M.; Zwart, F.M. - Psychoeducation of Partners of Bipolar-Manic Patients. J Affect Disord 21: 15-18, 1991.

Wulsin, L.; BaChop, M.; Hoffman, D. - Group Therapy in Manic Depressive Illness. Am J Psychother 42: 263-271, 1988.

Zaretsky, A.E.; Segal, ZV.; Gemar, M. - Cognitive Therapy for Bipolar Depression: a Pilot Study. Can J Psychiatry 44: 491-494, 1999. 